\title{
Angina During Hospitalization
}

National Cancer Institute

\section{Source}

National Cancer Institute. Angina During Hospitalization. NCI Thesaurus. Code C80479.

Clinical diagnosis of angina during a hospitalization, of which ang ina was not the admitting diagnosis. 